\title{
Management of Post-Operative Pain after Gyneco-Obstetrical Surgery: Practice of Transversus Abdominis Plane Bloc (Tap Block) Echoguide at the Ignace Deen National Hospital
}

\author{
Donamou Joseph1, Bangoura Almamy², Touré Abdoulaye ${ }^{2}$, Camara Amadou Yalla ${ }^{3}$, \\ Traoré Abdourahamane Dine'1, Camara M'Mah Lamine1', Dramé Boubacar Atigou'2, \\ Camara Mariama Mohamed ${ }^{2}$, Orou Yerima Therese ${ }^{1}$, Touré Aboubacar ${ }^{4 *}$ \\ ${ }^{1}$ Anesthesia-Intensive Care Unit, Donka National Hospital, Conakry, Guinea \\ ${ }^{2}$ Anesthesia-Intensive Care Unit, National Ignace Deen Hospital, Conakry, Guinea \\ ${ }^{3}$ Medico-Surgical Emergency Department, Donka National Hospital, Conakry, Guinea \\ ${ }^{4}$ General and Visceral Surgery Department, Ignace Deen National Hospital, Conakry, Guinea \\ Email: ^atfmamad@gmail.com
}

How to cite this paper: Joseph, D., Almamy, B., Abdoulaye, T., Yalla, C.A., Dine, T.A., Lamine, C.M., Atigou, D.B., Mohamed, C.M., Therese, O.Y. and Aboubacar, T. (2021) Management of Post-Operative Pain after Gyneco-Obstetrical Surgery: Practice of Transversus Abdominis Plane Bloc (Tap Block) Echoguide at the Ignace Deen National Hospital. Open Journal of Anesthesiology, 11, 316-324.

https://doi.org/10.4236/ojanes.2021.1110031

Received: July 22, 2021

Accepted: October 16, 2021

Published: October 19, 2021

Copyright $\odot 2021$ by author(s) and Scientific Research Publishing Inc. This work is licensed under the Creative Commons Attribution International License (CC BY 4.0).

http://creativecommons.org/licenses/by/4.0/

(c) (i) Open Access

\begin{abstract}
Objectives: To describe the practice of ultrasound-guided TAP in the management of postoperative pain after gyneco-obstetric surgery. Methods: This was a descriptive prospective study carried out at the Gynecology-Obstetrics department of the Ignace Deen National Hospital over a period of three (03) months from February 01, 2020 to April 31, 2020. Results: In total, we collected 95 patients. These patients had a mean age of 30 \pm 9.5 years. The ASA I class was the most represented with $76 \%$ of the cases and the cesarean was the most performed intervention. Regarding the assessment of the pain score by the simple verbal scale (SVE) postoperatively at rest, the mean SLE scores at $\mathrm{H} 6$ were $0.17 \pm 0.38$; at $\mathrm{H} 12$ of $1.15 \pm 0.62$; at $\mathrm{H} 24$ of $0.84 \pm 0.51$; at $\mathrm{H} 360.45 \pm 0.52$ and at $\mathrm{H} 480.09 \pm 0.29$. On mobilization, the mean pain scores were $0.77 \pm 0.51$ at H6, at $\mathrm{H} 121.89 \pm 0.61$; at $\mathrm{H} 24$ of $1.53 \pm 0.56$; at $\mathrm{H} 36$ of $1 \pm 0.29$ and at $\mathrm{H} 48$ of $0.82 \pm 0.44$. The majority of our patients (66.3\%) had a mobilization time of less than 24 hours. The mean length of stay was $3.1 \pm 1.3$ days and most patients (82\%) were satisfied with the management of their pain by ultrasound-guided TAP block. Conclusion: Ultrasound-guided TAP is an effective technique for the management of postoperative pain in gyneco-obstetrics surgery. Its integration in a context of multimodal analgesia could improve the management of postoperative pain in gynecological obstetrics.
\end{abstract}




\section{Keywords}

Postoperative Pain, TAP Block, Ultrasound Guidance, Gyneco-Obstetrics Surgery

\section{Introduction}

Obstetric and gynecological surgery is the cause of severe to maximum postoperative pain (PDO) during the first 48 hours after surgery. The persistence of this pain has consequences for the postoperative rehabilitation of patients [1]. The current management of PDO after this surgery is based on a multimodal approach combining morphine with non-morphine analgesics [2]. The use of opiates, in particular morphine, is a very popular alternative for the treatment of PDO in gyneco-obstetric surgery; however, the use of this is often associated with several side effects such as nausea, vomiting, pruritus, respiratory depression, etc. other analgesia methods such as abdominal wall blocks have been shown to be effective in managing this pain. Among these blocks, the transversus abdominis plane block (TAP block) occupies a special place [3]. This block allows anesthesia of the nerves of the anterior wall of the abdomen (territory T9-L1), obtained by infiltration of a local anesthetic solution in the transverse plane [3] [4]. A study carried out in India by Jadon A. et al. [5] in 2018 on the role of ultrasound-guided TAP block as a component of a multimodal analgesic regimen for cesarean sections showed that ultrasound-guided TAP block reduces the intensity of pain and decreases the need for additional opioid analgesics when it is used in the multimodal analgesia protocol for pain relief after obstetric surgery. Another study carried out in Gabon by Mandji L. et al. [2] in 2017 on the efficacy of ultrasound-guided transversus abdominis plane block in cesarean analgesia reported that ultrasound-guided TAP block provides quality analgesia after cesarean section and may be an attractive alternative to morphine. In Guinea, the literature remains limited on this subject. The objective of this study is to describe the practice of ultrasound-guided TAP in the multimodal analgesic management of PDO after gyneco-obstetric surgery in the Gynecology-Obstetrics department of the Ignace Deen National Hospital.

\section{Patients and Methods}

This was a prospective study of descriptive type covering a period of three (03) months from February 01, 2020 to April 31, 2020 and carried out at the Gynecology-Obstetrics department of the Ignace Deen National Hospital. As regards an investigation of internal practice, no authorization was requested; however, the patients were systematically informed. We included all the patients who had undergone an ultrasound-guided TAP block for emergency gyneco-obstetric surgery or scheduled under general anesthesia or spinal anesthesia without intrathecal morphine who agreed to participate in the study. We did not include 
patients who presented one or more of the following criteria:

- Refusal of patients to participate in the study.

- Infection at the puncture site.

- Morbid obesity (BMI $\left.>40 \mathrm{~kg} / \mathrm{m}^{2}\right)$.

- Allergy to local anesthetics.

\section{Protocol}

All the patients underwent a pre-anesthetic consultation and information on the ultrasound-guided TAP block was given before the date scheduled for the surgery. After welcoming and installing the patient on the operating table, cardiorespiratory monitoring (TA, SpO2, FC, FR) and a peripheral venous route were set up. The patients received either GA or spinal anesthesia (without morphine).

At the end of the operation (surgical closure and dressing), a bilateral ultrasound-guided TAP block with Ropivacaine $2 \mathrm{mg} / \mathrm{ml}(3 \mathrm{mg} / \mathrm{kg}$ ) without exceeding a dose of $200 \mathrm{mg}$ was performed under general anesthesia or under spinal anesthesia (without morphine) according to the approach known as "echuided" inter costo-iliac. Supine using an ECO 1 CHISON ultrasound system fitted with a $10 \mathrm{MHz}$ high frequency linear probe protected by a sterile plastic envelope. After rigorous asepsis of the puncture area, the ultrasound probe was placed at the level of the umbilicus, the probe was slid laterally to be positioned at the level of the middle axillary line midway between the iliac crest and the inferior border of the last rib while remaining on the horizontal line passing through the umbilicus; so as to successively visualize from the surface to the depth the following structures: The subcutaneous tissue, external oblique muscle (MOE), internal oblique muscle (MOI), transverse abdominal muscle (MTA), peritoneum, and intraperitoneal structures. Once the three muscle planes (MOE, MOI, MTA) were well individualized, a $100 \mathrm{~mm}$ Visioplex VYGON $20 \mathrm{G}$ needle with a short bevel was introduced into the plane $2 \mathrm{~cm}$ from the ultrasound probe. Then, under visual control, the progression of the needle was followed up to the level of the "transverse plane" located between the internal oblique muscle and the transverse muscle. When the tip of the needle is viewed in the transverse plane, Ropivacaine $2 \mathrm{mg} / \mathrm{ml}(3 \mathrm{mg} / \mathrm{kg})$ at a concentration adapted to the patient's weight was injected (without exceeding a dose of $200 \mathrm{mg}$ ); $100 \mathrm{mg}$ of Ropivacaine diluted in $20 \mathrm{cc}$ was injected sideways after an aspiration test eliminating the intravascular position of the needle. Diffusion of local anesthesia produced a detachment of the fascia in the form of a convex hypoechoic lens visible on ultrasound, driving the transverse muscle below and the internal oblique above. After performing the ultrasound-guided TAP block, the patients were transferred to SSPI. The analgesic protocol was multimodal in addition to the ultrasound-guided TAP block, the patients received paracetamol at a dosage of $1 \mathrm{~g}$ every 6 hours for 48 hours. No difficulty was encountered during the realization of the TAP block. Pain intensity was assessed at rest and on movement or coughing using the Simple Verbal Scale (SLE): This simple verbal scale is rated 
from 0 to $4: 0=$ no pain; $1=$ mild pain; $2=$ moderate pain $=; 3=$ severe pain; $4=$ unbearable pain.

The various scores were collected postoperatively at the sixth hour (H6), at the twelfth hour (H12), at the twenty-fourth hour (H24), at the thirty-sixth hour (H36) and at the forty-eighth hour (H48).

The data were collected on a pre-established survey form. These data were represented by socio-demographic characteristics (age and BMI), clinical (ASA class, anesthesia technique and type of intervention) and technical (time to perform the block, evaluation of post-pain. operative, resumption of walking, patient length of stay and patient satisfaction). The statistical analysis was carried out by the STATA 15 software. The qualitative data were expressed as a percentage. Quantitative data were expressed as mean with standard deviation.

\section{Results}

During the study we collected 95 patients. The 20 to 29 age group was the most represented with an average of $30 \pm 9.5$ years and extremes of 18 and 60 years. These patients had a mean BMI of $23.5 \pm 2.8 \mathrm{~kg} / \mathrm{m}^{2}$. Most of our patients (76\%) were classified ASA I. Cesarean section was the type of procedure most performed with $65.2 \%$ of cases. The majority (82\%) of patients received general anesthesia. The socio-demographic characteristics, the type of intervention and the type of anesthesia are summarized in Table 1.

The mean time to complete ultrasound-guided block TAP was $3.2 \pm 0.7 \mathrm{~min}$. Postoperatively, at rest, the mean EVS scores at $\mathrm{H} 6$ were $0.17 \pm 0.38$; at $\mathrm{H} 12$ of $1.15 \pm 0.62$; at $\mathrm{H} 24$ of $0.84 \pm 0.51$; at H36 $0.45 \pm 0.52$ and at H48 $0.09 \pm 0.29$. The mean resting EVS scores are summarized in Table 2. On mobilization, the mean scores were $0.77 \pm 0.51$ at $\mathrm{H} 6$, at $\mathrm{H} 121.89 \pm 0.61$; at $\mathrm{H} 24$ of $1.53 \pm 0.56$; at $\mathrm{H} 36$ of $1 \pm 0.29$ and at $\mathrm{H} 48$ of $0.82 \pm 0.44$. The mean EVS scores on mobilization are summarized in Table 3. The mobilization time was less than 24 hours for the majority (66.3\%) of the patients. The mean length of hospital stay was $3.1 \pm 1.3$ days. Most (82\%) of the patients were satisfied. We did not record any complications.

\section{Discussion}

Our results show that ultrasound-guided TAP block is an effective analgesic technique for the management of postoperative pain after gyneco-obstetric surgery. Indeed, gyneco-obstetric surgery is the source of intense pain during the first 48 hours postoperatively [6]. This pain is amplified by the fact that this surgery mainly concerns young women. These two criteria are described in the literature as predictive factors that would expose to more severe acute postoperative pain as well as to higher morphine consumption in the recovery room [7]. In our study, our population was young and female, therefore predisposed to present severe postoperative pain, however, the use of ultrasound-guided TAP block produced mild pain in our patients. This result could be explained by the good analgesia provided by the ultrasound-guided TAP block. The direct benefit of 
Table 1. Socio-demographic characteristics, type of intervention and type of anesthesia.

\begin{tabular}{|c|c|c|}
\hline Variables & Ultrasound-guided TAP $(n=95)$ & Percentage \\
\hline \multicolumn{3}{|l|}{ Age (years) } \\
\hline \multicolumn{3}{|c|}{ Mean \pm standard deviation $30 \pm 9.5$} \\
\hline$<20$ & 9 & 9.47 \\
\hline $20-29$ & 40 & 42.11 \\
\hline $30-39$ & 35 & 36.84 \\
\hline 40 years and over & 11 & 11.58 \\
\hline \multicolumn{3}{|l|}{ BMI $\left(\mathrm{kg} / \mathrm{m}^{2}\right)$} \\
\hline \multicolumn{3}{|c|}{ Mean \pm standard deviation $23.5 \pm 2.8$} \\
\hline 30 & 4 & 4 \\
\hline$\geq 30$ & 91 & 96 \\
\hline \multicolumn{3}{|l|}{ ASA } \\
\hline I & 72 & 75.79 \\
\hline II & 17 & 17.89 \\
\hline III & 6 & 6.32 \\
\hline \multicolumn{3}{|l|}{ Type of intervention } \\
\hline Cesarean & 62 & 65.26 \\
\hline Myomectomy & 29 & 30.53 \\
\hline Total hysterectomy & 2 & 2.11 \\
\hline Ectopic pregnancies & 2 & 2.11 \\
\hline Ovarian cystectomy & 1 & 1.05 \\
\hline \multicolumn{3}{|l|}{ Type of anesthesia } \\
\hline General anaesthesia & 78 & 82.11 \\
\hline Spinal anesthesia & 17 & 17.89 \\
\hline
\end{tabular}

Table 2. Means of EVS scores of patients at rest.

\begin{tabular}{cc}
\hline characteristics & Values \\
EVS rest at H6 & \\
Mean \pm standard deviation & $0.17 \pm 0.38$ \\
Median & 0 \\
Extreme & $0 ; 1$ \\
\hline EVS rest at H12 & \\
Mean \pm standard deviation & $1.15 \pm 0.62$ \\
Median & 1 \\
Extreme & $0 ; 3$ \\
EVS rest at H24 & \\
Mean \pm standard deviation & $0.84 \pm 0.51$ \\
Median & 1 \\
Extreme & $0 ; 2$ \\
\hline
\end{tabular}




\section{Continued}

\begin{tabular}{cc} 
EVS rest at H36 & \\
Mean \pm standard deviation & $0.45 \pm 0.52$ \\
Median & 0 \\
Extreme & $0 ; 2$ \\
\hline EVS rest at H48 & $0.09 \pm 0.29$ \\
Mean \pm standard deviation & 0 \\
Median & $0 ; 1$ \\
Extreme & \\
\hline
\end{tabular}

Table 3. Average EVS scores of patients on mobilization.

\begin{tabular}{|c|c|}
\hline characteristics & Values \\
\hline \multicolumn{2}{|l|}{ EVS mob at $\mathrm{H} 6$} \\
\hline Mean \pm standard deviation & $0.77 \pm 0.51$ \\
\hline Median & 1 \\
\hline Extreme & $0 ; 2$ \\
\hline \multicolumn{2}{|l|}{ EVS mob at $\mathrm{H} 12$} \\
\hline Mean \pm standard deviation & $1.89 \pm 0.61$ \\
\hline Median & 2 \\
\hline Extreme & $1 ; 3$ \\
\hline \multicolumn{2}{|l|}{ EVS mob 24 hours a day } \\
\hline Mean \pm standard deviation & $1.53 \pm 0.56$ \\
\hline Median & 1 \\
\hline Extreme & $1 ; 3$ \\
\hline \multicolumn{2}{|l|}{ EVS mob at $\mathrm{H} 36$} \\
\hline Mean \pm standard deviation & $1 \pm 0.29$ \\
\hline Median & 1 \\
\hline Extreme & $0 ; 2$ \\
\hline \multicolumn{2}{|l|}{ EVS mob at $\mathrm{H} 48$} \\
\hline Mean \pm standard deviation & $0.82 \pm 0.44$ \\
\hline Median & 1 \\
\hline Extreme & $0 ; 2$ \\
\hline
\end{tabular}

ultrasound-guided TAP block is the reduction in the intensity of postoperative pain both at rest and during movement when it is used in the multimodal analgesia protocol [8] [9]. This observation is found in our study where, whatever the time at rest or during mobilization, the postoperative pain in patients who received ultrasound-guided TAP block was mild. The direct benefit of ultrasound-guided TAP block is the reduction in the intensity of postoperative pain both at rest and during movement when it is used in the multimodal analgesia protocol [8] [9]. This observation is found in our study where, whatever the time at rest or during mobilization, the postoperative pain in patients who received 
ultrasound-guided TAP block was mild. The direct benefit of ultrasound-guided TAP block is the reduction in the intensity of postoperative pain both at rest and during movement when it is used in the multimodal analgesia protocol [8] [9]. This observation is found in our study where, whatever the time at rest or during mobilization, the postoperative pain in patients who received ultrasound-guided TAP block was mild. TAP bloc provides good quality analgesia for the two components of postoperative pain (parietal and visceral pain) after gyneco-obstetrical surgery. It acts much more on the parietal component of the post-operative pain and makes it possible to obtain an excellent analgesia at the level of the latter; however, according to studies, the inhibition of nociceptive impulses at this level has a beneficial role on the visceral component of pain [3]. Currently, the success rate of TAP block has improved a lot due to the use of ultrasound. The difficulties in performing this block using the anatomical method were therefore resolved by providing ultrasound. This makes it possible to correctly identify the anatomical structures, to visualize the transverse plane and to check the correct position of the needle. It also gives an idea of the precise location of the injected product and therefore better chances of success of the block [10]. All of our blocks were performed under ultrasound, which explains their success and the good analgesia found in all of our patients.

This efficacy of ultrasound-guided TAP block on postoperative pain found in our study has been the subject of several publications. In a meta-analysis examining the effect of ultrasound-guided TAP block, it was concluded that the technique provides analgesia comparable to that of opioids and reduces their consumption without the side effects of the latter [11]. In another study, Tugba Karaman et al. in 2018 in Turkey showed that ultrasound-guided TAP decreases postoperative pain scores, reduces opioid consumption and improves patient comfort after total hysterectomy [12]. Hyun-Jung Shin et al. in 2011 in South Korea showed in their study that TAP block significantly reduced pain scores at rest and on exertion after gynecological surgery [13]. An African study carried out by Mandji L et al. in 2017 in Gabon reported that ultrasound-guided block TAP provides quality analgesia after cesarean section and may be an attractive alternative to morphine [2]. Thus, we see that the ultrasound-guided block TAP occupies an important place in the therapeutic arsenal for the management of postoperative pain by being integrated into the concept of multimodal analgesia. This efficiency of the ultrasound-guided TAP block was also reflected in a reduced postoperative mobilization time, a shortening of the hospital stay and a high level of satisfaction found in our patients. Regarding the mobilization time, it usually varies from 3 to 4 days in our countries [14]. In our series, the patients who benefited from the ultrasound-guided TAP block had for the most part a mobilization time of less than 24 hours. This reduced mobilization time was found in the study by Malhotra et al. in 2005 in India, which showed that their patients wandered more quickly at 3 p.m. [15]. The low intensity of postoperative pain at rest and especially during exercise could explain this result. As for the length of hospitalization, it was shortened in our study compared to the 
length of hospitalization usually observed in patients who underwent gyneco-obstetric surgery, which is 5 to 7 days depending on the type of surgery [14]. In our study, we found a shorter hospital stay of less than 4 days in the majority of patients who benefited from ultrasound-guided TAP block. These results could be explained by the use of the ultrasound-guided TAP block which allowed good management of postoperative pain, which is one of the main factors which prolongs patients' hospital stay. Satisfaction is a subjective fact, it depends on several factors which are the good progress of the surgical intervention and the good management of the postoperative pain. In our series, we noted a high level of satisfaction in most of the patients. Our results are similar to those of Bengono R et al. in 2016 in Cameroon who found in their study a high satisfaction rate of $89.3 \%$ in patients who benefited from the ultrasound-guided TAP block [16]. Our study has limitations insofar as it was evaluated on a small sample. Also the factors that could affect the pain have not been studied which would have helped identify factors.

\section{Conclusion}

Our study has shown that the ultrasound-guided TAP block can be perfectly adapted in the multimodal analgesia protocol for the management of postoperative pain after gyneco-obstetric surgery. The use of ultrasound facilitated the realization of the TAP block and guaranteed its success. Its integration in a context of multimodal analgesia could improve the management of postoperative pain in gynecology and obstetrics.

\section{Conflicts of Interest}

The authors declare no conflicts of interest regarding the publication of this paper.

\section{References}

[1] Yapo, P. (2018) Postoperative Pain: Contributing Factors and Management. RAMUR (Special Congress), 23, 147-150. https://doi.org/10.2307/j.ctv1gbrwc4.14

[2] Mandji, L.J., Edjo, N.G., Matsanga, A., Okoue, O.R., Sougou, M.E., Tchoua, R., et al. (2017) Efficacy of Ultrasound Transverse Abdominal Plan Block in Analgesia for Cesarean Section. African Journal of Anesthesiology and Emergency Medicine, 1, 14-19.

[3] Donamou, J., Touré, A., Touré, M.K., Keita, S. and Keita, N. (2017) Postoperative Analgesia after Enlarged Colpohysterectomy with Lymphadenectomy: Comparison of Intravenous Morphine versus Transversus Abdominalis Plane Bloc at Donka CHU National Hospital in Conakry. African Journal of Anesthesiology and Emergency Medicine, 2, 35-39.

[4] Sammos, G., Rn, C. and Chey, W.R. (2015) Use of Transversus Abdominis Plane (TAP) Blocks for Pain Management in Elderly Surgical Patients. AORN Journal, 102, 493-497. https://doi.org/10.1016/j.aorn.2015.09.003

[5] Ashok, J., Jain, P., Chakraborty, S., et al. (2018) Role of Ultrasound Guided Transversus Abdominis Plane Block as a Component of Multimodal Analgesic Regimen 
for Lower Segment Caesarean Section: A Randomized Double Blind Clinical Study. BMC Anesthesiology, 18, 53. https://doi.org/10.1186/s12871-018-0512-x

[6] (1998) Consensus Conference on Postoperative Pain in Adults and Children. Annales Françaises d Anesthesie et de Réanimation, 17, 445-461.

[7] Kehlet, H., Jensen, T.S. and Woolf, C.J. (2006) Persistent Postsurgical Pain: Risk Factors and Prevention. The Lancet, 367, 1618-1625. https://doi.org/10.1016/S0140-6736(06)68700-X

[8] McDonnell, J.G., Curley, G., Carney, J., Benton, A., Costello, J., Maharaj, C.H., et al. (2008) The Analgesic Efficacy of Transversus Abdominis Plane Block after Caesarean Delivery: A Randomized Controlled Trial. Anesthesia \& Analgesia, 106, 186-191. https://doi.org/10.1213/01.ane.0000290294.64090.f3

[9] McMorrow, R.C., Mhuircheartaigh, R.J., Ahmed, K.A., Aslani, A., Ng, S.C., Conrick, M.I., et al. (2011) Comparison of Transversus Abdominis Plane Block vs Spinal Morphine for Pain Relief after Caesarean Section. British Journal of Anaesthesia, 106, 706-712. https://doi.org/10.1093/bja/aer061

[10] Hebbard, P., et al. (2007) Ultrasound-Guided Transversus Abdominis Plane (TAP) Block. Anaesthesia and Intensive Care, 35, 616-617.

[11] Siddiqui, M.R.S., Sajid, M.S., David, R., Uncles, M.B., Cheek, L. and Baig, M.K. (2011) A Meta-Analysis on the Clinical Effectiveness of Transversus Abdominis Plane Block. Journal of Clinical Anesthesia, 23, 7-14.

https://doi.org/10.1016/j.jclinane.2010.05.008

[12] Karamana, T., Asker, Z.O., Karamana, S., et al. (2018) The Effects of Transversus Abdominis Plane Block on Analgesic and Anesthetic Consumption during Total Abdominal Hysterectomy: A Randomized Controlled Study. Revista Brasileira de Anestesiologia, 68, 285-291. https://doi.org/10.1016/j.bjane.2018.01.002

[13] Shin, H.-J., et al. (2011) Preemptive Analgesic Efficacy of Ultrasound-Guided Transversus Abdominis Plane Block in Patients Undergoing Gynecologic Surgery via a Transverse Lower Abdominal Skin Incision. Korean Journal of Anesthesiology, 61, 413-418. https://doi.org/10.4097/kjae.2011.61.5.413

[14] Rafanomezantsoa, T.A., Rakotondrainibe, A., Rasoamampianina, L.E., et al. (2016) Early Rehabilitation after Cesarean Operation at the CHU of Obstetric Gynecology Befelatanana. Journal of Anesthesia-Resuscitation, Emergency Medicine and Toxicology, 8, 37-40.

[15] Malhotra, N., Khanna, S., Pasrija, S., Jain, M. and Agarwala, R.B. (2005) Early Oral Hydration and Its Impact on Bowel Activity after Elective Caesarean Section-Our Experience. European Journal of Obstetrics \& Gynecology and Reproductive Biology, 120, 53-56. https://doi.org/10.1016/j.ejogrb.2004.08.009

[16] Bengono, R.B., Amengle, A.L., Jemea, B., Mbengono, J.A., Mekolo, D., Etound, P.O., et al. (2016) Interest of the Transversus Abdominis Plane Bloc (TAP) Surgical in the Management of Analgesia after Cesarean Section at the Central Hospital of Yaoundé. Journal of Medicine and Pharmacy, 6, 571-577. 